\title{
Ultrasonographic Diagnosis of an Isolated Cardiac Metastasis in a Patient with High-Grade Gastrointestinal Stromal Tumor
}

\author{
Bartolomucci Francesco $^{1}$, Giuseppe Nasso ${ }^{2 *}$, Cipriani Francesco ${ }^{1}$, Esposito Gianpiero ${ }^{3}$, \\ Valenti Giovanni $^{1}$, Giuseppe Speziale ${ }^{2}$ \\ ${ }^{1}$ Division of Cardiology, L. Bonomo Hospital, Andria, Italy \\ ${ }^{2}$ Division of Cardiac Surgery, Anthea Hospital, GVM Care \& Research, Bari, Italy \\ ${ }^{3}$ Division of Cardiac Surgery, Santa Maria Hospital, Bari, Italy \\ Email: "gnasso@libero.it
}

Received January 24, 2012; revised March 29, 2012; accepted April 28, 2012

\begin{abstract}
Aims: Secondary cardiac tumours are rare conditions, often difficult to suspect. We aim to describe the diagnostic potential of transthoracic and transesophageal echocardiography in this field, and to discuss their reliability as modalities of choice for preoperative diagnosis. Methods and Results: We used several complementary diagnostic approaches: Color-Doppler transthoracic echocardiography, transesophageal echocardiography, regional myocardial perfusion test and three-dimensional transthoracic echocardiography. The latter was employed to confirm the previously performed tests. All tests revealed the presence of an echogenic, oval-shaped and well-delimited mass in the basal part of intervenetricular septum. Conclusion: Even though it was initially asymptomatic and characterized by scarce clinical manifestations, the intra-cardiac mass was reliably identified in this patient by means of the cited diagnostic modalities, which also allowed adequate surgical planning. The immunohistochemical examination revealed the secondary nature of this tumour. Basic and advanced echocardiography examinations were crucial in the decision-making process.
\end{abstract}

Keywords: Echocardiography; GIST; Cardiac Tumours; Three-Dimensional Echocardiography

\section{Case Report}

A 54-year-old man with mild hypertension was scheduled for echocardiography due to a second degree systolic murmur heard at the cardiac apex during a pre-endoscopy cardiologic assessment. Blood tests were unremarkable. Five years earlier, the patient had undergone surgical treatment of an oesophageal neoplasm recognized, later identified as a gastrointestinal stromal tumor (GIST). Postoperatively, the patient had received adjuvant treatment with Imatinib (Gleevec). Both total-body computed tomography (CT) and upper gastrointestinal endoscopy were performed annually and neither recurrence nor repetitive lesions were identified at any time. Color-Doppler transthoracic echocardiography (TTE) was performed using a Philips IE33 scanner; it showed normal dimensions of the cardiac chambers, a normal global and segmental left ventricular function and marked intervenetricular septal hypertrophy (Figure 1). Subsequent transesophageal echocardiography identified an echogenic mass within the basal portion of the interventricular sep-

${ }^{*}$ Corresponding author. tum (Figure 2). Therefore, regional myocardial perfusion test was performed (intravenous contrast agent: SonoVue $1 \mathrm{ml}$ in bolus, then $0.8 \mathrm{ml}$ per minute during the examination) revealed a normal myocardial blood supply and confirmed the presence of a well-delimited, ovalshaped mass within the interventricular septum. The existence of this mass was also confirmed by three-dimensional transthoracic echocardiography (Figure 3). The

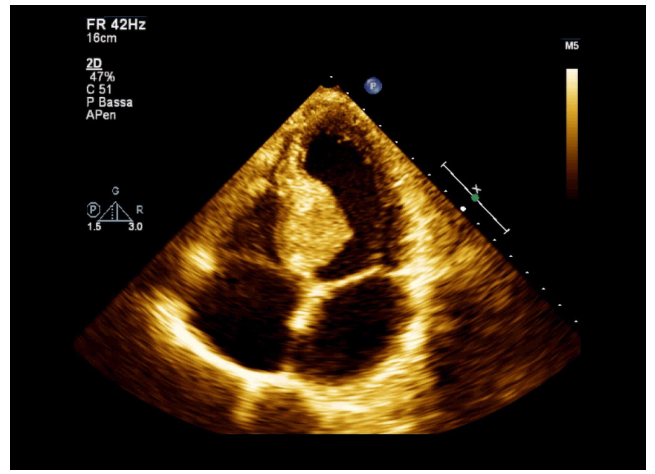

Figure 1. Two-dimensional transthoracic echocardiography image showing septal hypertrophy. 


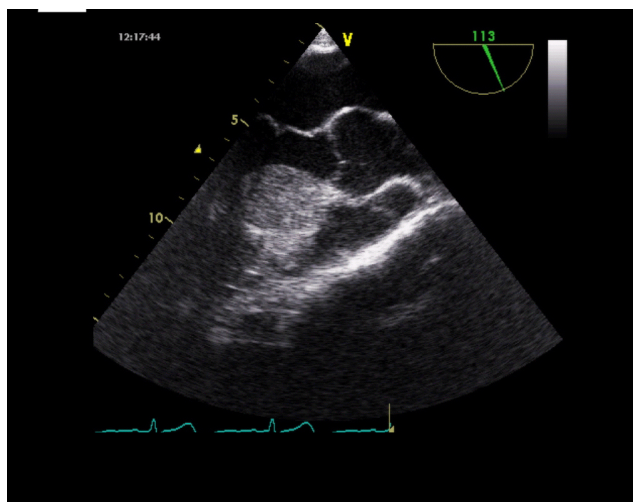

Figure 2. Transesophageal echocardiography image showing an echogenic mass within the basal portion of the interventricular septum.

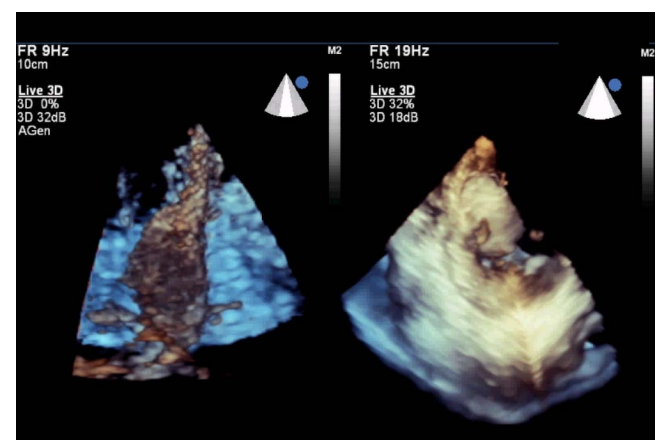

Figure 3. Three-dimensional transthoracic echocardiogramphy: confirmation of the presence of the mass within the basal septum.

lesion was surgically excised; the immunohistochemical analysis revealed neoplastic cells positive for desmin, synaptophysin, cromogranine and CD-117 (c-kit) immunophenotype. These findings allowed to define the metastatic nature of the lesion (sub-endocardial locally infiltrating multi-nodular GIST). After an uneventful recovery (Figure 4), the patient was discharged and started post-excisional chemotherapy with GLEEVEC and Sandostatin. In February 2010 a spiral CT scan showed again a $4 \times 4.8 \mathrm{~cm}$ mass within the interventricular septum. At the abdominal level, two nodules within the left lobe of the liver ( $5 \mathrm{~cm}$ and $2 \mathrm{~cm}$ wide) were identified, along with another $1.5 \mathrm{~cm}$ nodule within the right kidney. Transthoracic Echocardiography confirmed the presence and the dimension of the intraseptal cardiac mass. Following surgical excision of the largest abdominal mass, two-dimensional TTE (Figure 5) and TTE perfusion imaging (Figure 6) showed a much wider intraseptal mass, with markedly hypoperfused areas, likely due to necrosis. The decision to perform a new intracardiac excision was then taken. Our patient underwent repeat median resternotomy. Under extracorporeal circulation, aortotomy was performed and the endoventricular mass (2.2 $\times 3 \mathrm{~cm}$ ) was visualized through the aortic valve. The

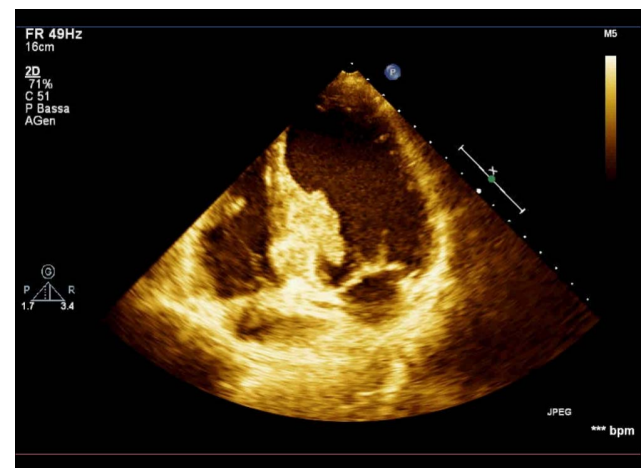

Figure 4. Post-excisional transthoracic echocardiography.

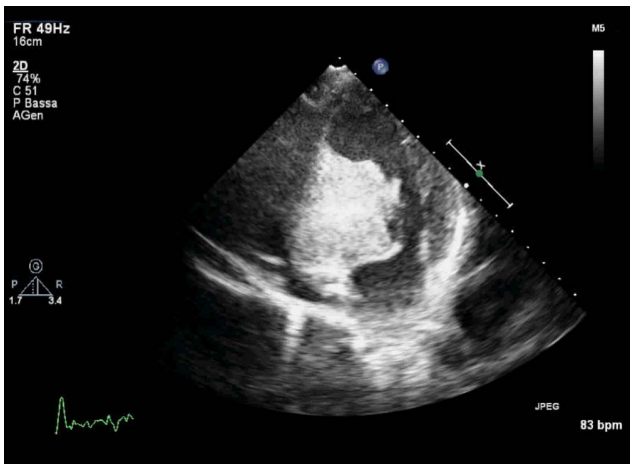

Figure 5. Two-dimensional transthoracic echocardiography image showing recurrence within the basal septum at followup.

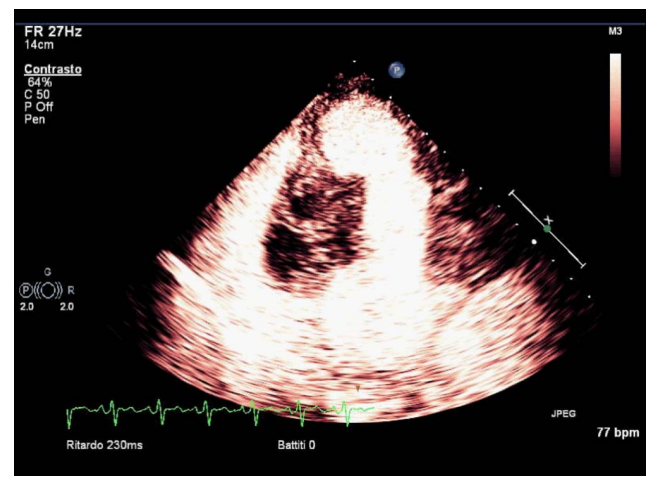

Figure 6. Transthoracic echocardiography perfusion image identifying hypoperfused areas within the mass.

mass infiltrated the interventricular septum, and could be excised with a portion of surrounding tissue. The conesquent interventricular defect was closed with a PTFE patch using a combined trans-aortic and a trans-tricuspid access. The patient was weaned from extracorporeal circulation and prolonged surgical hemostasis was required. Twenty-four hours after the intervention, surgical revision for bleeding was necessary, without identification of any surgical source of bleeding. During this procedure a progressive worsening of the cardiac function caused the patient's death. 


\section{Discussion}

Secondary cardiac tumors are rare, with an incidence of $4 \%$ in non-selected samples and up to $20 \%$ in post-mortem analyses of patients deceased for the evolution of a malignant neoplasm [1]. Signs and symptoms are nonspecific, as in our case. This makes difficult the noninvasive diagnosis. Nonetheless, patients may present with ventricular arrhythmias and/or signs of congestive heart failure depending on the location of the lesion, thus prompting echocardiography assessment. Few previous cases reported over metastatic intracardiac lesions in patients with oesophageal cancer [2,3]. We believe that in patients with history of malignant tumor and appearance of unexplained arrhythmias or heart failure, a high level of suspicion should be maintained with respect to the possibility of intracardiac metastasis.

Transthoracic and transesophageal echocardiography may be useful for the diagnostic process of such masses [4]. In particular, TTE is effective as a screening modality, while transesophageal echocardiography provides an accurate estimation of lesion dimension. Such estimation, as illustrated by our case, is at least comparable to that obtained by CT scan. Additionally, three-dimensional TTE provides an excellent visualization of the relationship of the mass with the surrounding structures, thus allowing precise operative planning and the assessment of the potential for surgical resection. However, the poor prognosis of these patients should be kept in mind during the decision-making process.

\section{Conclusion}

The integration of basic and advanced echocardiography modalities (Color-Doppler transthoracic echocardiogramphy, transesophageal echocardiography, regional myocardial perfusion test and three-dimensional transthoracic echocardiography) are invaluable in the diagnosis and preoperative evaluation of patients affected by intracardiac metastatic lesions.

\section{REFERENCES}

[1] J. Butany, V. Nair, A. Naseemuddin, G. M. Nair, C. Catton and T. Yau, "Cardiac Tumours: Diagnosis and Management," The Lancet Oncology, Vol. 6, 2005, No. 4, pp. 219-228. doi:10.1016/S1470-2045(05)70093-0

[2] M. Kataoka, K. Shigemitsu, S. Tanabe, T. Ohara, T. Takahata and S. Nose, "Sudden Death from Metastatic Esophageal Cancer to the Ventricular Septum," Japanese Journal of Thoracic and Cardiovascular Surgery, Vol. 53, No. 7, 2005, pp. 365-368. doi:10.1007/s11748-005-0051-5

[3] M. Maeda, T. Goto, M. Harigai, T. Itoh, T. Moriki and T. Miyashita, "Myocardial Metastasis from Squamous Cell Carcinoma of the Esophagus," General Thoracic and Cardiovascular Surgery, Vol. 57, No. 8, 2009, pp. 440-445. doi:10.1007/s11748-009-0408-2

[4] M. N. Sheppard and R. Mohiaddin, "Tumors of the Heart," Future Cardiology, Vol. 6, No. 2, 2010, pp. 181-193. doi:10.2217/fca.09.62 